\title{
Spatio-temporal structure of a poloidal Alfvén wave detected by Cluster adjacent to the dayside plasmapause
}

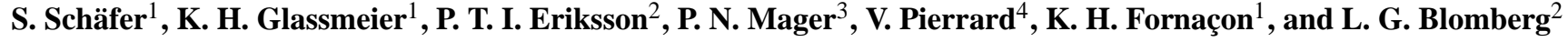 \\ ${ }^{1}$ Institut für Geophysik und extraterrestrische Physik, TU Braunschweig, Germany \\ ${ }^{2}$ Space and Plasma Physics, School of Electrical Engineering, Royal Institute of Technology Stockholm, Sweden \\ ${ }^{3}$ Inst. of Solar-Terrestrial Physics (ISTP), State Academy of Science, Siberian Branch, Irkutsk, P.O. Box 291, 664033, Russia \\ ${ }^{4}$ Belgian Institute for Space Aeronomy, Brussels, Belgium
}

Received: 5 February 2008 - Revised: 28 April 2008 - Accepted: 16 May 2008 - Published: 30 June 2008

\begin{abstract}
A case study of a poloidal ULF pulsation near the dayside plasmapause is presented based on Cluster observations of magnetic and electric fields. The pulsation is detected close to the magnetic equatorial plane at $\mathrm{L}$ shells $L=[4.4,4.6]$ and oscillates with a frequency of $f=23 \mathrm{mHz}$. Investigating the wave energy flux reveals the standing wave nature of the observed pulsation. An estimation of the azimuthal wave number exposes a narrow azimuthal structure of the wave field with $m \approx 160$. Spatial and temporal characteristics of the pulsation are analyzed in detail by representing data in a field line related coordinate system and a rangetime-intensity representation. This allows an estimation of both the spatial extension of the wave field in the radial direction and its temporal decay rate. The analysis furthermore indicates that the same field lines are excited to a standing wave oscillation twice. Furthermore an accurate identification of a phase jump of the wave field across $\mathrm{L}$ shells is possible. Comparing the radial localization of the detected wave with theoretically expected field line eigenfrequencies reveals that the wave field is confined in the Alfvén resonator at the outer edge of the plasmapause.
\end{abstract}

Keywords. Magnetospheric physics (Magnetosphereionosphere interactions; MHD waves and instabilities; Plasmasphere)

\section{Introduction}

A wide variety of magnetohydrodynamic waves occur in magnetospheric regions of the Earth excited by different pro-

Correspondence to: S. Schäfer

(seb.schaefer@tu-bs.de) cesses inside the magnetosphere and the solar wind. Some of these processes excite standing waves at magnetospheric field lines in the ultra low frequency (ULF) range between $1 \mathrm{mHz}$ and $100 \mathrm{mHz}$. In general these standing waves are divided into two different wave modes, toroidal and poloidal oscillations.

Toroidal oscillations are believed to be driven by e.g. solar wind impulses (Allan et al., 1986) and Kelvin-Helmholtz instabilities at the magnetopause (Fujita et al., 1996; Engebretson et al., 1998) generating a compressional wave which propagates across magnetic field lines. Due to the process known as field line resonance (Tamao, 1965; Southwood, 1974), the compressional wave can be transformed to an Alfvén wave propagating parallel to the magnetic field. Wave reflection at the ionosphere can lead to the formation of a standing wave at a specific field line. Toroidally oscillating field lines are perturbed in the azimuthal direction, i.e. field magnitude and plasma density are not changed by this wave.

Poloidal oscillations of magnetic field lines derive their energy by plasma instabilities inside the magnetosphere such as drift mirror (Hasegawa, 1969) and bounce instability (Southwood et al., 1969; Southwood and Kivelson, 1982; Glassmeier et al., 1999). In contrast to the toroidal mode, poloidal oscillations are perturbed in the radial and field aligned direction causing a compression of the magnetic field. When the compressive perturbation of the magnetic is small compared to the radial one, poloidal oscillations are usually referred as poloidal Alfvén waves.

Both types of wave modes are prevalently observed and discussed using spacecraft and ground based measurements. The latter method allows long duration observations of field lines excited by ULF waves (e.g. Samson et al., 1971; Walker et al., 1979). However, amplitude and polarization of ULF

Published by Copernicus Publications on behalf of the European Geosciences Union. 
waves observed at the ground are modified by the ionosphere (Hughes, 1974; Hughes and Southwood, 1976; Glassmeier, 1984) and consequently, modelling of the ionospheric screening effect is necessary for an accurate understanding of the ground based observations (e.g. Sciffer et al., 2004, 2005; Glassmeier, 1988).

In contrast to ground observations spacecraft observations provide direct insight into the structure of field line oscillations (e.g. Singer and Kivelson, 1979; Singer et al., 1982; Engebretson and Cahill, 1981; Engebretson et al., 1986). However, single spacecraft observations have a limitation as they exhibit an ambiguity between spatial and temporal variations. A multi-spacecraft mission is not affected by this limitation and hence the four Cluster spacecraft (Escoubet et al., 1997) are suitable to investigate both, the spatial and temporal structure of ULF pulsations as shown in recent studies (e.g. Eriksson et al., 2005; Schäfer et al., 2007).

Spatial and temporal characteristics of toroidal ULF wave activity strongly depends on the plasma background conditions and the generation mechanism. Mode coupling of an incoming fast magnetosonic wave to an Alfvén wave occurs at magnetic field lines where the field parallel component of the phase velocity of the compressional wave matches the local Alfvén velocity $v_{A}=B / \sqrt{2 \mu_{0} \rho}$ that is influenced by local magnetic field strength $B$ and plasma mass density $\rho$. When additionally the eigenfrequency of the field line complies with the frequency of the fast mode, the coupling between both wave modes becomes resonant. This process is known as field line resonance, which appears also in dipolar magnetic field configuration (e.g. Chen and Cowley, 1989). The spatial structure of the resulting toroidally polarized, standing Alfvén wave is expected to show an almost Gaussian amplitude variation in radial direction, i.e. across L shells, accompanied with a $180^{\circ}$ change in the wave phase around the resonant magnetic field line (Southwood, 1974). Both spatial features were detected in ULF pulsations by ground magnetometer and ionospheric observations, respectively (e.g. Walker et al., 1979; Glassmeier et al., 1984a). The field line resonance process is most efficient for small azimuthal wave numbers $m \sim 1$ (e.g. Kivelson and Southwood, 1986; Lee and Lysak, 1990), i.e. the toroidal wave field is expected to exhibit a large spatial structure in the azimuthal direction. The lifetime of resonant field line oscillations is between several minutes and more than one hour limited due to wave damping at the ionosphere, where the wave energy dissipates due to Joule heating (e.g. Glassmeier et al., 1984b).

Understanding the spatio-temporal structure of poloidal Alfvén waves with $m \gg 1$ (e.g. Allan et al., 1983) requires detailed knowledge on the poloidal eigenfrequency $\Omega_{P}$ of magnetospheric field lines. While the toroidal eigenfrequency $\Omega_{T}$ is calculated from the travel time of an Alfvén wave along the field line (Warner and Orr, 1979), $\Omega_{P}$ is additionally affected by field line curvature (e.g. Leonovich and Mazur, 1990, 1993), finite plasma $\beta$ and external currents, such as the ring current (Klimushkin, 1998b; Klimushkin et al., 2004). The spatial and temporal properties of a poloidal wave field are affected by the difference between the field line eigenfrequencies, $\Omega_{P}$ and $\Omega_{T}$. The radial wave number of Alfvénic perturbations in this case is given by (e.g. Leonovich and Mazur, 1993; Klimushkin, 2000)

$k_{r}^{2} \propto \frac{\omega^{2}-\Omega_{P}^{2}}{\Omega_{T}^{2}-\omega^{2}}$,

where $\omega$ is the wave frequency.

Wave propagation in radial direction is possible where $k_{r}^{2}>0$. This region is called the transparent region. At the poloidal surface $L_{P}$, where $\omega=\Omega_{P}$, we have $k_{r}=0$, i.e. this surface represents a reflection boundary in radial direction. The toroidal surface $L_{T}$ with $\omega=\Omega_{T}$ is characterized by $k_{r} \rightarrow \infty$. This surface can be interpreted as that magnetic shell, where resonance takes place and the poloidal perturbation is converted to a mainly toroidal one. Thus, two different cases need to be considered (e.g. Klimushkin et al., 2004).

First, for $\Omega_{P}$ and $\Omega_{T}$ varying monotonically across magnetic $\mathrm{L}$ shells, a poloidal Alfvén wave can be generated near the poloidal surface $L_{P}$. During its temporal evolution the wave propagates radially towards the toroidal surface $L_{T}$, where the wave can be characterized as an Alfvén resonance. The radial structure of the wave field changes in time from a Gaussian-like distribution at the beginning to a complex and small-scaled structure at the end of the lifetime. Simultaneously, the wave polarization changes from poloidal to toroidal. An explanation for this spatio-temporal behavior can also be given by considering phase mixing effects on field lines excited independently and oscillating with their own eigenfrequencies (e.g. Mann and Wright, 1995).

The suggested temporal change in wave polarization is not reported until now. The reason for this lacking observation is probably the rapid damping of the poloidal oscillation due to ionospheric damping, which implies that the transformation from poloidal to toroidal is incomplete (e.g. Klimushkin and Mager, 2004). In general this kind of wave evolution mechanism is predicted to occur preferably where the field line eigenfrequencies $\Omega_{P}$ and $\Omega_{T}$ decrease monotonically across magnetic L shells. The outcome of this is a standing Alfvén wave trapped radially between $L_{T}$ and $L_{P}$.

As a second possibility a cavity for poloidal Alfvén waves can be formed if the wave field is confined by two poloidal turning points $L_{P}$, i.e. at local extreme values of $\Omega_{P}$ (e.g. Vetoulis and Chen, 1994, 1996; Leonovich and Mazur, 1995; Denton and Vetoulis, 1998; Klimushkin, 1998a). This particular condition is satisfied at the inner and outer edge of the plasmapause, where the radial distribution of plasma density reaches a minimum and maximum, respectively. Mager and Klimushkin (2006) have modeled the spatio-temporal structure of a standing Alfvén wave in such a wave resonator assuming an impulse wave source. For a fundamental harmonic structure along the field line $(N=1)$ they predict a radial structure of the wave field comparable to a Gaussian 


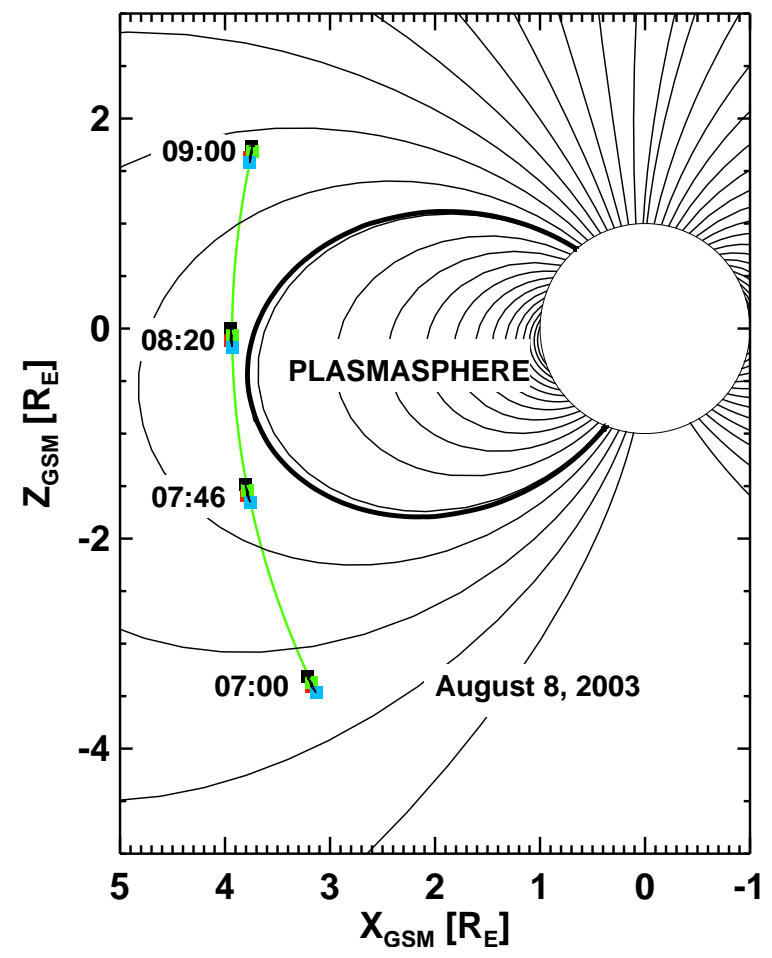

Fig. 1. The Cluster orbits in the dayside magnetosphere on $8 \mathrm{Au}-$ gust 2003, 07:00 and 09:00 UT. The ULF pulsation investigated is detected between 07:46 and 08:20 UT. The thick black line marks the plasmapause at $L=4.23 R_{E}$.

and a constant poloidal wave polarization. By contrast, the radial structure of the second longitudinal harmonic $(N=2)$ develops soon after the impulse from a Gaussian distribution across L shells to smaller radial scales, similar to the radially propagating wave discussed above. Observations of poloidal Alfvén waves usually reveal a second harmonic structure of the oscillation along the field line (e.g. Takahashi and Anderson, 1992). Furthermore the radial localization of the wave field requires a change in the wave phase by $180^{\circ}$ across $\mathrm{L}$ shells (Klimushkin et al., 2004).

ULF wave fields exhibit a rather complex spatial structure, as described above. This structure also determines the temporal evolution of these waves as well as their polarization. Therefore, checking the theoretically derived concepts requires a detailed spatio-temporal analysis of the ULF wave field. An ideal tool for this analysis is the Cluster mission with its four spacecraft. As the perigees of the polar orbits are at around $4 R_{E}$ the satellites frequently cross the region near the inner or outer edge of the plasmapause which is commonly localized between 3 and $6 R_{E}$ depending on the solar wind activity. Consequently, ULF wave activity can be detected regularly in this region of interest near the extreme values of poloidal and toroidal field line eigenfrequencies.

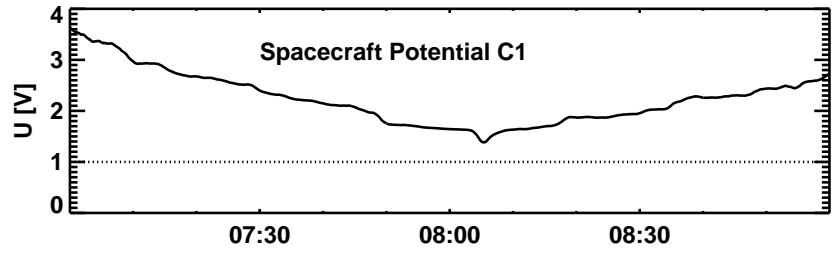

Fig. 2. Spacecraft potential measured by Cluster spacecraft $\mathrm{C} 1$. Values of $U<1 \mathrm{~V}$ (dotted line) are expected inside the plasmasphere.

The present case study concentrates on the detailed analysis of a standing poloidal field line oscillation detected in the vicinity of the dayside plasmapause. Fluxgate magnetometer (FGM) measurements (Balogh et al., 2001) and electric field observations of the EFW instrument Gustafsson et al. (2001) onboard Cluster are used. The investigation of the spatiotemporal structure of the observed wave field is favored by a Cluster configuration with spacecraft separation of the order of $0.2 R_{E}$.

\section{Observations}

During the time interval 8 August 2003, between 07:46 and 08:20 UT, the Cluster satellites have traversed the dayside magnetosphere close to the plasmapause and have detected a poloidally polarized ULF pulsation oscillating with a nearly monochromatic frequency of $f_{\mathrm{obs}}=23 \mathrm{mHz}$. Figure 1 shows the orbits of all four spacecraft during the time interval discussed together with field lines modeled using the Tsyganenko magnetospheric magnetic field model (Tsyganenko, 1995; Tsyganenko and Stern, 1996). The extent of the satellite tetrahedron is only around $1000 \mathrm{~km}$.

The position of the plasmapause is determined using the dynamical simulations of plasmapause formation developed by Pierrard and Lemaire (2004) and Pierrard and Cabrera (2005). For the given time interval we determine a radial distance of the plasmapause in the magnetic equatorial plane of $4.23 R_{E}$. The field line with the corresponding vertex is assumed as the plasmapause out of the equatorial plane and is indicated by the thick black line in Fig. 1. The orbits of the Cluster spacecraft approach the plasmapause, but the boundary is not crossed by the satellites as shown by the measurements of the spacecraft potential $U$ (Fig. 2). As the potential $U$ is inversely proportional to the plasma electron density $n_{e}$, one would expect a clear change in $U$ during the crossing of the plasmapause due to the large differences in the plasma density between plasmapause and outer magnetosphere. Furthermore a spacecraft potential of $U \approx 1 \mathrm{~V}$ is expected inside the plasmasphere (e.g. Gustafsson et al., 2001). Both indications are not present in the time series of $U$ (Fig. 2).

Magnetic and electric field data used are 4-s averages transformed into a Mean-Field-Aligned (MFA) coordinate 

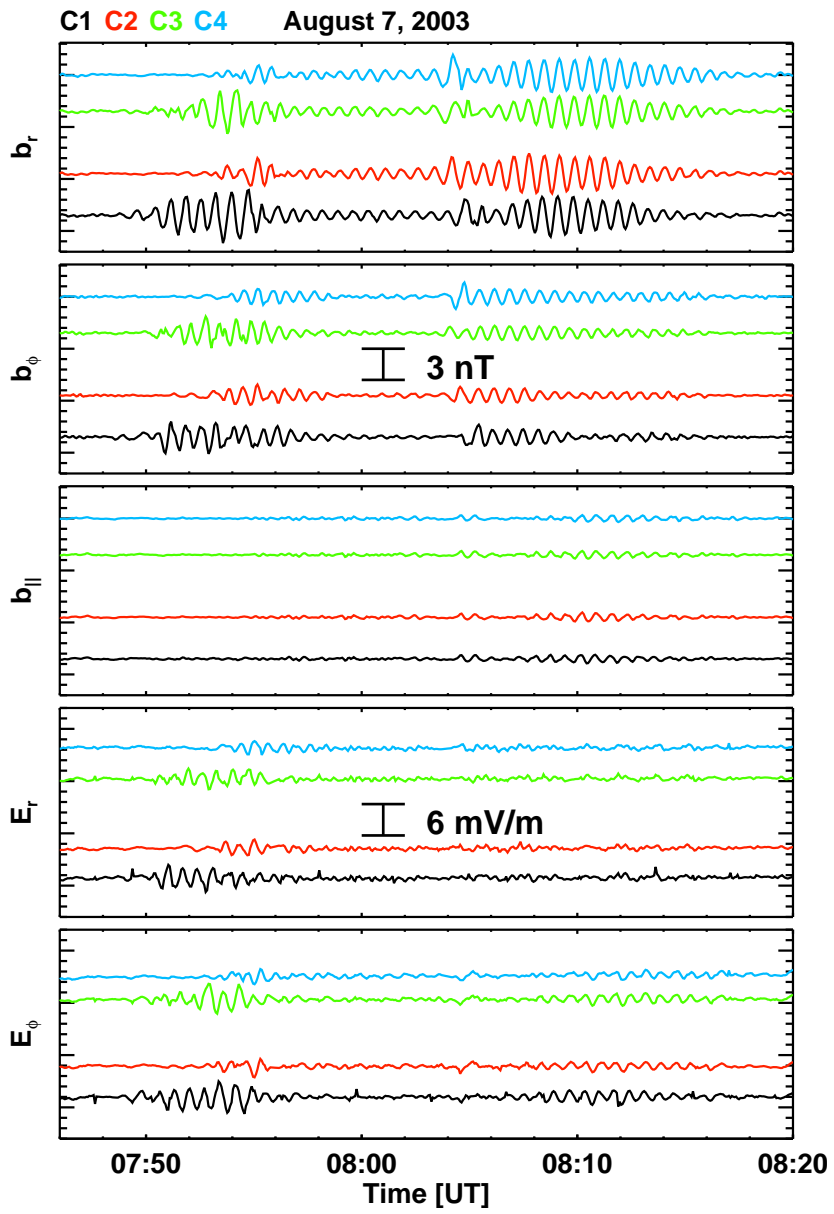

Fig. 3. FGM and EFW measurements for the four Cluster spacecraft (C1: black, C2: red, C3, green, C4: blue), transformed into a Mean-Field-Aligned coordinate system.

system, where the unit vector $\boldsymbol{e}_{\|}$is directed parallel to the background magnetic field $\boldsymbol{B}$. The unit vector in the azimuthal direction is $\boldsymbol{e}_{\phi} \propto \boldsymbol{e}_{\|} \times \boldsymbol{r}_{s c}$ and the radial unit vector $\boldsymbol{e}_{r} \propto \boldsymbol{e}_{\phi} \times \boldsymbol{e}_{\|}$, where $\boldsymbol{r}_{s c}$ is the position vector of the spacecraft. Figure 3 shows observations of the magnetic and electric fields from all four Cluster spacecraft. The first three panels exhibit the magnetic field components, $b_{r}, b_{\phi}$ and $b_{\|}$. The latter component has been defined by subtracting the mean magnetic field from the field aligned component to identify perturbations parallel to $\boldsymbol{B}$. The lower two panels display the electric field components $E_{r}$ and $E_{\phi}$, respectively. To describe the electric field perturbations we assume that $\boldsymbol{E} \cdot \boldsymbol{B}=0$, that is no field-aligned electric field component exists.

The pulsation event observed can be subdivided into two different wave packets with respect to its amplitude pattern (Fig. 3). The first packet occurs between 07:50 and 07:56, where amplitudes up to $2 \mathrm{nT}$ are observed in the $b_{r}$ component and up to $1 \mathrm{nT}$ in the $b_{\phi}$ component. The electric field oscillates mainly in the $E_{r}$ component with amplitudes up to
$2 \mathrm{mV} / \mathrm{m}$ at $\mathrm{C} 1$ and lower amplitudes at the other spacecraft.

The onset of the pulsation occurs at different times at different locations with spacecraft $\mathrm{C} 1$ detecting the perturbation first followed by spacecraft C3, C2 and C4. Furthermore, the amplitude pattern of both, the magnetic and electric fields are clearly different at each spacecraft, where distinct signals are seen in the $b_{r}$ and $E_{r}$ components of $\mathrm{C} 1$ and $\mathrm{C} 3$, that is those spacecraft observing the pulsation first.

The second wave packet is seen between 08:04 and 08:16, where again amplitudes up to $2 \mathrm{nT}$ are observed in $b_{r}$ and up to $1 \mathrm{nT}$ in $b_{\phi}$. In contrast to the first wave packet the oscillations in the $b_{r}$ components are regular at each spacecraft. The onsets of the wave are again different at the different spacecraft. But now the order has changed compared to the first packet. The pulsation event is first observed by $\mathrm{C} 2$ and $\mathrm{C} 4$ almost simultaneously followed by $\mathrm{C} 1$ and $\mathrm{C} 3$ observing the event about one minute later. The amplitude of the electric field is much smaller than in the first packet.

The Poynting vector $\boldsymbol{S}_{p}=\frac{1}{\mu_{0}} \boldsymbol{E} \times \boldsymbol{b}$ and the time integrated energy flux

$\left\langle\boldsymbol{S}_{p}\right\rangle=\frac{1}{T} \int_{0}^{T} \boldsymbol{S}_{p} d t$

provide further information about the observed signal. In our case the wave period is $T=43.5 \mathrm{~s}$. A standing wave is indicated by $\left\langle\boldsymbol{S}_{p}\right\rangle=0$ and an oscillating field parallel component of the Poynting vector $\boldsymbol{S}_{p, \|}$ (Chi and Russell, 1998). We found $\boldsymbol{S}_{p, r} \approx 0$ and $\boldsymbol{S}_{p, \phi} \approx 0$ (not shown here) and oscillations in $S_{p, \|}$ with amplitudes larger than field parallel component of the time integrated energy flux (Fig. 4) justifying $\left\langle S_{p, \|}\right\rangle \approx 0$ for the entire time interval. Accordingly, one can conclude that a standing field line oscillation is detected simultaneously by all four Cluster satellites. Furthermore both wave packets are observed close to the field line null point that is defined as the position along the oscillating field line where $\left\langle\boldsymbol{S}_{p}\right\rangle=0$ (Allan, 1982; Ozeke et al., 2005). Assuming that the ionospheric conductivity is similar at northern and southern footprint of the field line the null point corresponds to the point where the field line intersects the magnetic equatorial plane.

\section{Analysis and interpretation}

\subsection{Temporal evolution}

The simultaneous measurements of the four Cluster satellites allows in principle the investigation of spatial and temporal structures of the detected ULF pulsation, but it requires a data representation in four dimensions. An approach to this representation is a field line related coordinate system, the L-D-M coordinate system, introduced by Schäfer et al. (2007). The coordinate L resembles the McIlwain parameter (McIlwain, 1966) describing the vertex of a specific field line with respect to the center of the Earth in units of $R_{E}$. The second coordinate $\mathrm{D}$ is defined as the distance between the spacecraft 


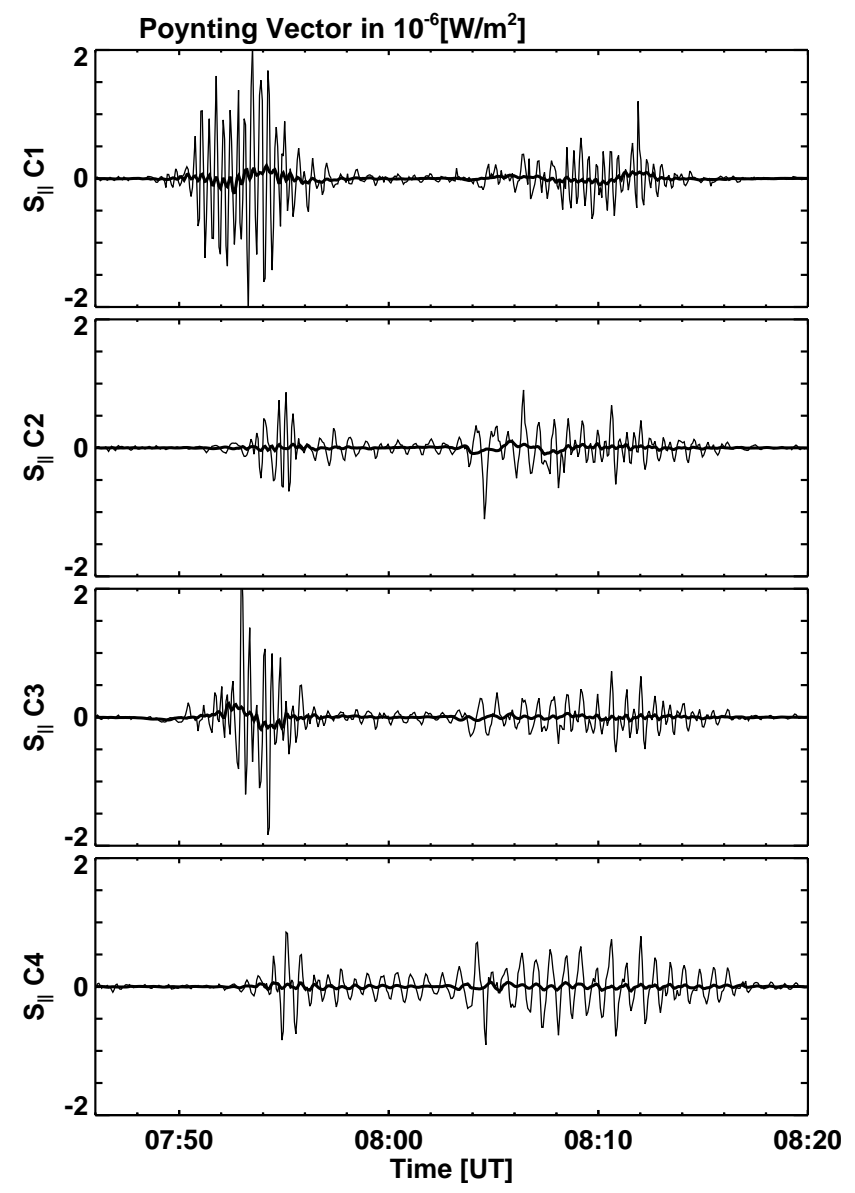

Fig. 4. Field parallel components of the Poynting vector $\boldsymbol{S}_{p}$ observed by Cluster spacecraft. The thick line represents the time averaged energy flux.

and the field line vertex along the field line. The third coordinate $M$ is equivalent to the magnetic local time (MLT) at which the measurements are taken. During the time interval analyzed the spacecraft orbits vary in the $\mathrm{M}$ coordinate between $M=13.72$ and $M=13.84$ (Fig. 5), which implies that the Cluster satellites cover a spatial range of $0.05 R_{E}$ in azimuthal direction.

The orbits of the four satellites are displayed in Fig. 6 transformed to the L-D coordinates, where the exact position of each spacecraft is marked at four specific times $t_{1}=07: 54: 02, t_{2}=07: 55: 06, t_{3}=08: 04: 10$ and $t_{4}=08: 09: 26 \mathrm{UT}$. The satellites move from the Southern magnetic Hemisphere $D<0 R_{E}$ across the magnetic equator $D=0 R_{E}$ into the Northern Hemisphere $D>0 R_{E}$. Between 07:46 and 08:20 UT all four Cluster spacecraft cross field lines with $\mathrm{L}$ values between 4.4 and 4.8. The color index of each orbit is related to the transverse amplitude $A=\left(A_{r}^{2}+A_{\phi}^{2}\right)^{0.5}$ to identify regions of high wave activity. Here $A_{r}$ and $A_{\phi}$ are the instantaneous amplitudes of the poloidal and toroidal magnetic field component, respectively,

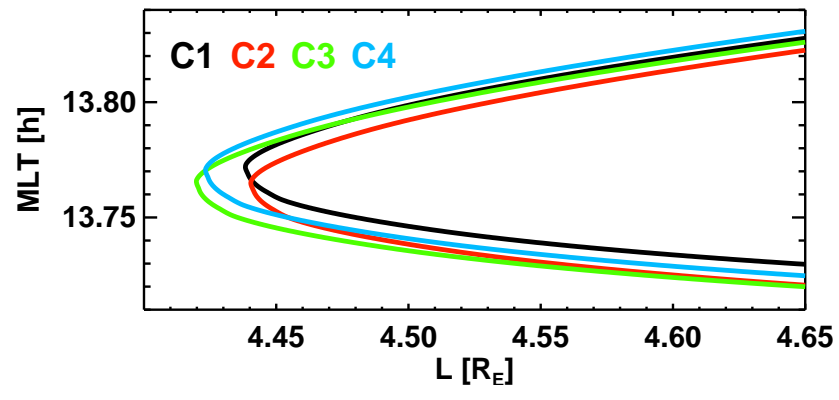

Fig. 5. Magnetic local time of Cluster orbit plotted against the $\mathrm{L}$ coordinate.

determined using the analytic signal or Carson-Gabor representation of the time series $b_{r}$ and $b_{\phi}$ (e.g. Glassmeier, 1980). Figure 7 displays a more detailed description of the instantaneous transverse amplitude $A$.

The L-D representation reveals, that the first maximum occurs when crossing field lines in the range $L=[4.50,4.65]$ in the southern magnetosphere at $D \approx-0.6 R_{E}$, whereas the second maximum occurs crossing different field lines at $L=[4.42,4.50]$ close to the field line vertex, i.e. close to the magnetic equator. The existence of an amplitude maximum in $b_{r}$ together with the absence of any electric field oscillations near the magnetic equator suggest the existence of an even mode oscillation. This assumption is consistent with earlier studies, where dayside Pc4 pulsations have been interpreted as second harmonic wave modes (e.g. Singer et al., 1982; Takahashi and Anderson, 1992).

Now we describe further details of the detected wave field by discussing the four selected times $t_{1}, \ldots, t_{4}$ marked in Figs. 6 and 7. At $t_{1}$ an amplitude maximum of around 2.6 nT is observed in spacecraft $\mathrm{C} 1$ and $\mathrm{C} 3$ simultaneously when both satellites are located nearly at the same $\mathrm{L}$ shell $L=4.57$. At the same time spacecraft $\mathrm{C} 2$ and $\mathrm{C} 4$ are located about $0.05 R_{E}$ further out, where no clear amplitude maxima are visible. Accordingly, the radial extension of the first wave packet can be estimated at time $t_{1}$; we found a width of around $w=0.1 R_{E}$.

At $t_{2}$ spacecraft $\mathrm{C} 2$ and $\mathrm{C} 4$ detects maxima with amplitudes of $A=1.9 \mathrm{nT}$ (C2) and $A=1.2 \mathrm{nT}$ (C4). These peaks are found at $\mathrm{L}$ shell $L=4.59$, close to the field lines where $\mathrm{C} 1$ and $\mathrm{C} 3$ detect maxima. Consequently the amplitude of the field line oscillation decreases between $t_{1}$ and $t_{2}$. Assuming that the amplitude of the signal decays exponentially in time, i.e. $A(t) \propto e^{-\gamma t}$, the decay constant $\gamma$ can be determined from

$\gamma=\frac{1}{\Delta t} \ln \left(\frac{A\left(t_{1}\right)}{A\left(t_{2}\right)}\right)$

with $\Delta t=64 \mathrm{~s}$. Amplitude values are obtained from spacecraft $\mathrm{C} 3$ at $t_{1}$ and $\mathrm{C} 2$ at $t_{2}$, as both spacecraft have the same field parallel position $D=-0.58 R_{E}$ at these times. Inserting 


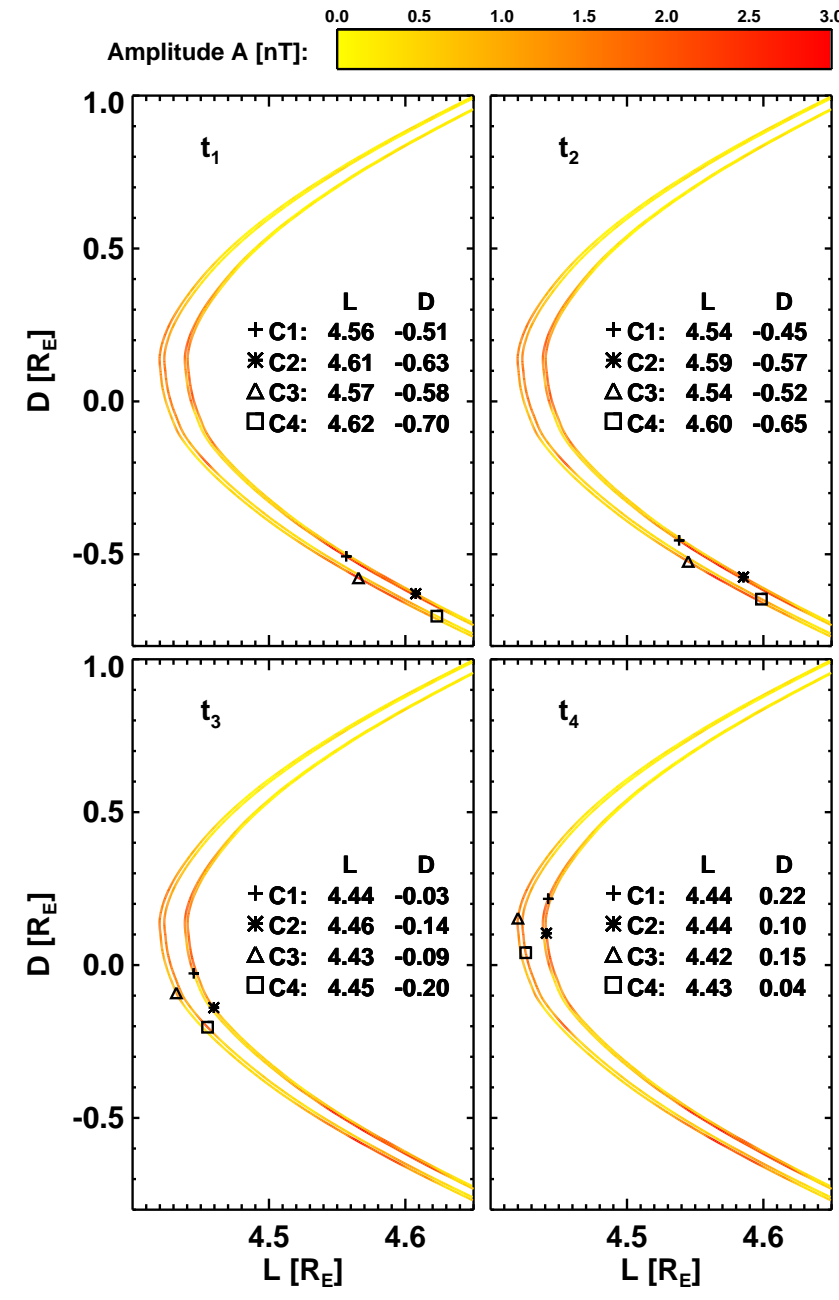

Fig. 6. Cluster orbits in L-D coordinates at $t_{1}-t_{4}$. The spacecraft move from negative to positive $\mathrm{D}$ values across the magnetic equator $\left(D=0 R_{E}\right)$. The color index gives the instantaneous amplitude of the transverse magnetic field oscillation.

$A\left(t_{1}\right)=2.6 \mathrm{nT}$ and $A\left(t_{2}\right)=1.8 \mathrm{nT}$ in Eq. (3) leads to a decay constant of $\gamma \approx 5 \mathrm{mHz}$ and a corresponding e-folding time $1 / \gamma$ giving an approximation of the lifetime $\tau=2 / \gamma \approx 400 \mathrm{~s}$. The amplitude pattern observed by the four Cluster satellites at two different times indicates the existence of a transient standing field line oscillation at $\mathrm{L}$ shells $L=[4.5,4.65]$.

The field line oscillation does not disappear after time $t_{2}$ despite the detected amplitude decay: While the satellites move to inner $\mathrm{L}$ shells and towards the magnetic equator spacecraft $\mathrm{C} 2, \mathrm{C} 3$ and $\mathrm{C} 4$ detect an amplitude peak simultaneously at $t_{3}$, whereas a peak is visible at $\mathrm{C} 1$ around $40 \mathrm{~s}$ later. Comparing with time series of $b_{r}$ (Fig. 3) shows that these maxima mark the onset of the second wave packet detected at $\mathrm{L}$ shells $L=[4.42,4.50]$. This wave packet is fully developed at time $t_{4}$ in all four spacecraft observations.
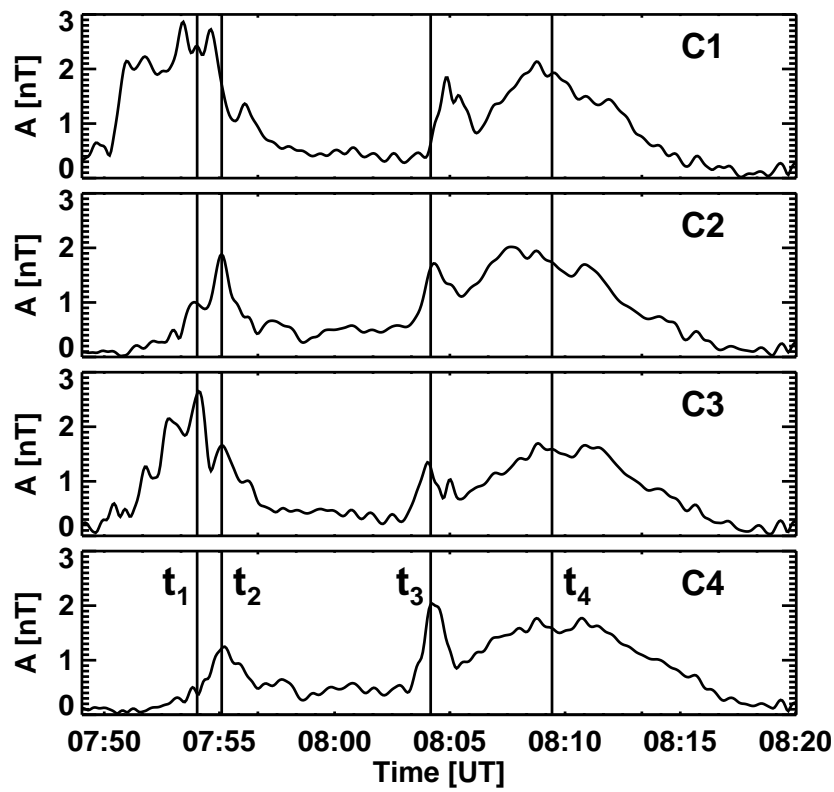

Fig. 7. Instantaneous amplitude $A(t)$ of the transverse magnetic field oscillation.

The observed temporal evolution of the wave amplitude justifies assuming that both wave packets are excited successively. The first wave is detected at the end of its lifetime, whereas the second wave packet can be understood as another wave excited at the same magnetic field lines. As discussed in Sect. 1 the wave polarization of an ULF pulsation is expected to change from poloidal to toroidal during the temporal evolution of the wave field. Comparing the ratio of the toroidal amplitude $A_{\phi}$ and the poloidal amplitude $A_{r}$ of both wave packets (Fig. 3) reveals that the first one is characterized by $A_{\phi} \approx A_{r}$, i.e. the toroidal part of the amplitude is comparable to the poloidal part. In contrast to that the second wave packet exhibits $A_{\phi}<A_{r}$, i.e. it is "less" toroidal than the first wave packet. Following the theory the observed pattern of the wave polarization indicates that the first wave packet is detected in a later phase of the temporal evolution than the second wave packet. Consequently the double excitation of magnetic field lines within the same region is a possible scenario to describe the observed amplitude pattern and wave polarization.

\subsection{Azimuthal wave number}

A typical feature of poloidal Alfvén waves is a small-scale spatial structure associated with a large azimuthal wave number $m$. The knowledge of the phase difference $\Delta \Psi$ of signals observed by two different satellites allows an estimation of this number:

$m=\frac{\Delta \Psi}{\Delta \Phi}=\frac{360^{\circ}}{\Delta \Phi} \frac{\Delta t}{T}$, 
where $\Delta \Phi$ is the spatial separation between the two satellites in the azimuthal direction, $\Delta t$ the time difference between points of the same wave phase and $T$ the wave period (e.g. Eriksson et al., 2005; Schäfer et al., 2007). A variation of the magnetic field $b \propto \exp (i m \Psi)$ is assumed. High resolution magnetometer data $(10 \mathrm{~Hz})$ are used to estimate the phase difference in the $b_{\phi}$ component of two satellites observed between 08:09 and 08:11 UT, where the data are bandpass filtered between $10 \mathrm{mHz}$ and $50 \mathrm{mHz}$ (Fig. 8).

During this time interval spacecraft $\mathrm{C} 1$ and $\mathrm{C} 2$ are located on the same L shell, $L=4.44$ (see Fig. 6). The angle in the azimuthal direction between the two satellites is $\Delta \Phi=0.26^{\circ} \pm 0.01^{\circ}$. The time difference between extreme values of the signals is $\Delta t=4.8 \mathrm{~s}$ which corresponds to a phase difference of $\Delta \Psi=40^{\circ}$ assuming a wave period of $T=1 / f_{\text {obs }}=43 \mathrm{~s}$. Assuming an uncertainty in $\Delta t$ of $\delta t=0.2 \mathrm{~s}$ due to the temporal resolution of the time series and in $T$ of $\delta T=1 \mathrm{~s}$ we have $m=155 \pm 11$, which is in good agreement with the theoretically expected high- $m$ values for poloidal ULF pulsations.

\subsection{Radial structure}

A range-time-intensity (RTI) representation as known from ground-based ULF wave observations (e.g. Walker et al., 1979; McDiarmid and Allan, 1990; Fenrich et al., 1995) is used to infer the radial structure and is displayed for the $b_{r}$ component in Fig. 9. The actual perturbations of the magnetic field measured at the same time have been linearly interpolated between the four spacecraft positions For the first wave packet, located at $\mathrm{L}$ shells between $L=4.50$ and $L=4.65$, the RTI plot reveals a clear spatial and temporal structure with perturbation maxima and minima moving across L-shells towards the Earth. The structured change from positive to negative perturbations indicates a clear phase change in the L-direction. The black line in the inset of Fig. 9 traces the zero of magnetic field amplitude as the wave field goes through its temporal cycle within a spatial structure that exhibits a phase jump in the radial direction. Due to the time variation imposed on such a spatial structure the wave phase moves apparently towards smaller L shells (Wright et al., 1999). This virtual Earthward moving phase structure is reminiscent of poleward moving bands of irregularities observed in ionospheric data associated with field line resonance (Walker et al., 1979; Poulter et al., 1982).

In contrast to the first wave packet the second one found at $L=[4.42,4.50]$ is composed of field lines oscillating exactly in phase (Fig. 9). Assuming that both wave packets originate from the oscillation of the same bundle of field lines and the phase jump is fixed between $L=4.50$ and $L=4.65$, the identification of a phase jump in the second wave packet is impossible. The Cluster satellites move parallel to the apparent position of the phase jump while detecting the second wave packet (see Fig. 6). This assumption is supported by the observation of the instantaneous amplitude $A$ at time $t_{4}$ :

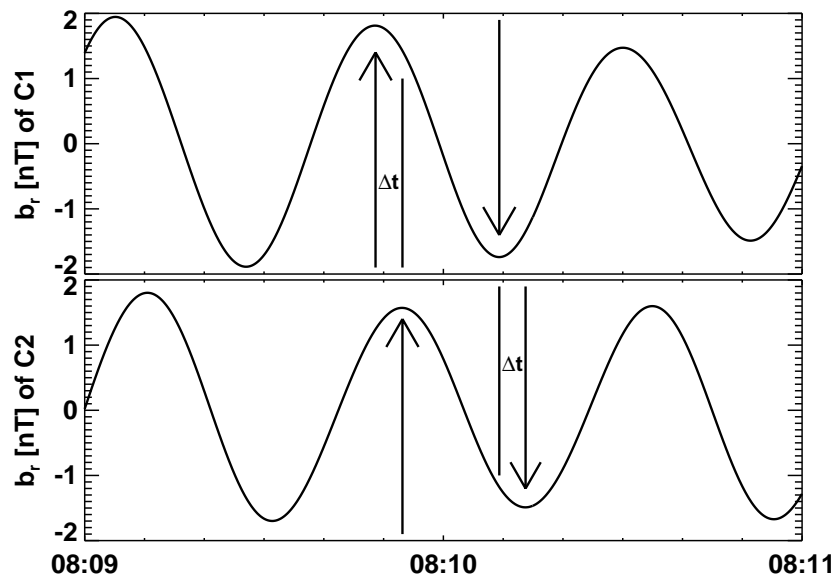

Fig. 8. Time series of the radial magnetic field oscillation of spacecraft $\mathrm{C} 1$ and $\mathrm{C} 2$.

Spacecraft $\mathrm{C} 1$ and $\mathrm{C} 2$ located at $L=4.45$ detects higher amplitude values, $A \approx 2.0 \mathrm{nT}$, than $\mathrm{C} 3$ and $\mathrm{C} 4$ located at $L=4.43$ detecting $A \approx 1.6 \mathrm{nT}$ (Fig. 7).

\subsection{A model}

For a more detailed understanding of the observed spatiotemporal structure we have modelled the ULF wave event using the following assumptions and observations:

(a) The pulsation is composed of two consecutively excited wave packets with lifetimes in the order of $\tau \approx 400 \mathrm{~s}$.

(b) The pulsation is a second longitudinal harmonic standing field line oscillation.

(c) The azimuthal wave number of the wave field is $m=155$.

(d) The wave phase changes by $180^{\circ}$ in the range of $\mathrm{L}$ shells $L \in[4,50,4.65]$.

The spatio-temporal variation is described by the following expression:

$b_{r, \text { model }}=b_{0}(t) \cdot S(D, t) \cdot B(\Psi) \cdot C(L)$,

where the function $b_{0}(t)$ represents the time dependent amplitude, $S(D, t)$ the standing wave character, $B(\Psi)$ the azimuthal and $C(L)$ the radial structure of the wave field. In the following we discuss each of these functions whose parameter range is varied restricted by the assumptions given above in order to fit the actual observed data simultaneously in all four Cluster satellites.

The time dependent amplitude $b_{0}(t)$ is given by two Gauss-functions generating the temporal development of the two wave packets:

$b_{0}(t)=b_{1} \exp \left(-\frac{\left(t-t_{1}\right)^{2}}{2 \tau_{1}^{2}}\right)+b_{2} \exp \left(-\frac{\left(t-t_{2}\right)^{2}}{2 \tau_{2}^{2}}\right)$. 


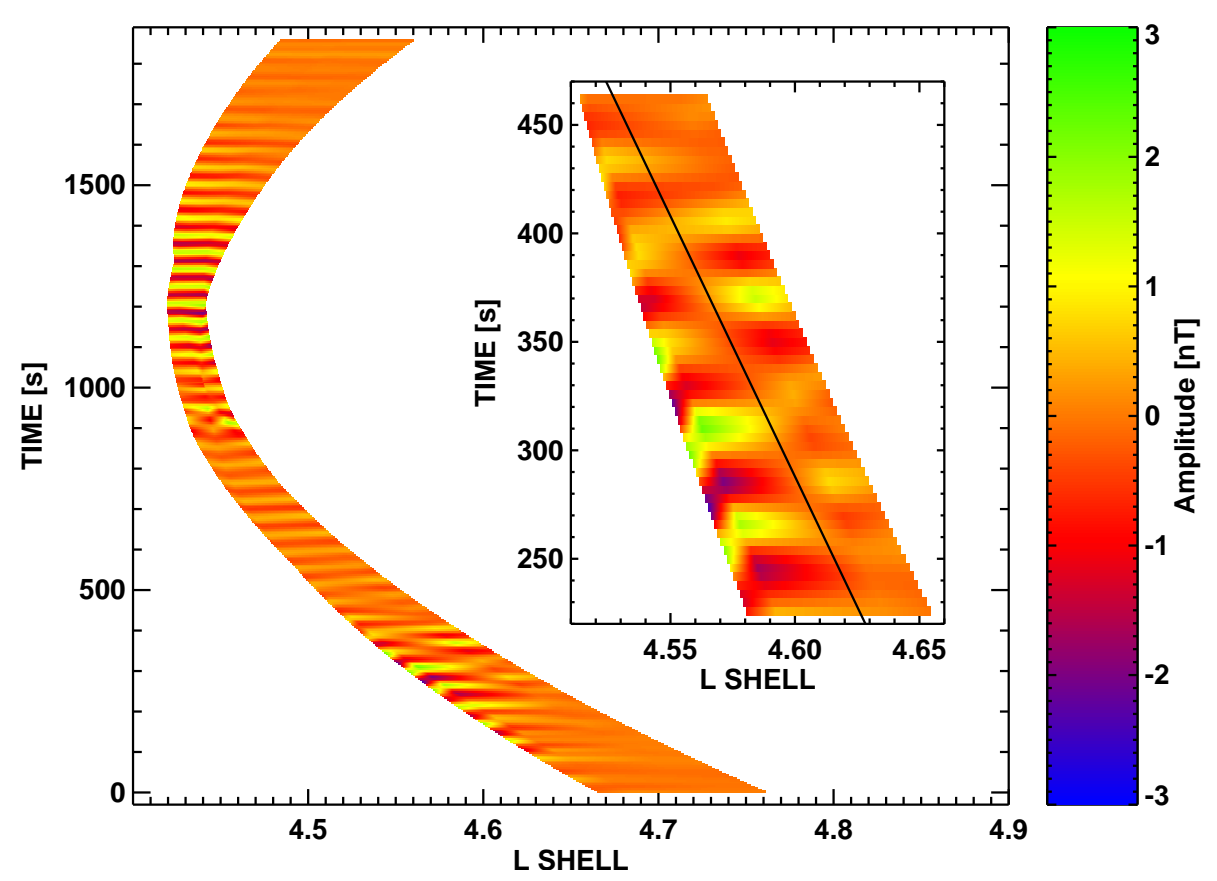

Fig. 9. Range-time-intensity plot of the 7 August 2003, 07:49-08:16 UT magnetic field fluctuations of the $b_{r}$ component. The ordinate is the time axis with $T=0$ s corresponding to 07:49 UT. The black line in the inlet corresponds to zero of wave amplitude indicating the apparent phase motion.

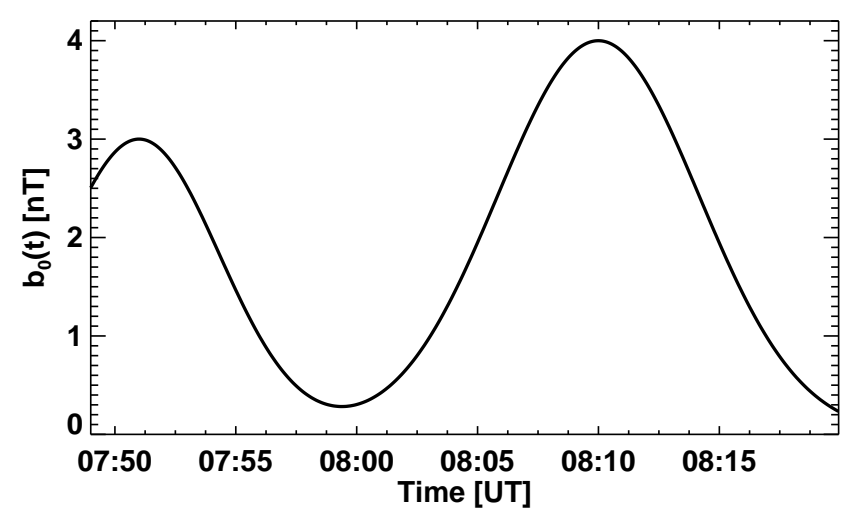

Fig. 10. Temporal development of the wave amplitude $b_{0}(t)$

The amplitude maxima in time are at $t_{1}=07: 51 \mathrm{UT}$ and $t_{2}=08: 10$ UT. For the first wave packet the maximum amplitude is $b_{1}=3.0 \mathrm{nT}$ and the width of the temporal amplitude distribution $\tau_{1}=200 \mathrm{~s}$. For the second wave packet we choose $b_{2}=4.0 \mathrm{nT}$ and $\tau_{2}=250 \mathrm{~s}$. The resulting temporal evolution of $b_{0}(t)$ is displayed in Fig. 10.

The standing wave along the magnetic field line is expressed by

$S(D, t)=\exp \left(i \omega t+i k_{\|} D\right)+\exp \left(i \omega t-i k_{\|} D\right)$,

where $k_{\|}$denotes the field parallel wave number. The cor- responding wavelength $\lambda_{\|}=2 \pi / k_{\|}$depends on the length of the field line $l$ and the harmonic number $N$ of the oscillation: $\lambda_{\|}=2 l / N$. The length of the field line with the vertices $L_{\text {pos }}=4.56$ is $l=10.4 R_{E}$. Assuming a second harmonic oscillation, as discussed above, results in a wavelength of $\lambda_{\|}=10.4 R_{E}$ and a field parallel wave number of $k_{\|}=0.096 R_{E}^{-1}$.

The azimuthal variation of the wave field is given by

$B(\Psi)=\exp (i m \Psi)$,

where an azimuthal wave number of $m=155$ is inserted which fits to the observed values of $m$. The corresponding azimuthal wavelength is $\lambda_{\Psi}=2 \pi / k_{\Psi}$ with $k_{\Psi}=m / L_{\text {pos }}=34.0 R_{E}^{-1}$. Accordingly, the azimuthal spatial scale of the wave is $\lambda_{\Psi}=0.18 R_{E}$.

The transverse structure of the wave field is described by a Gauss function and a phase function representing the amplitude variation and the change of the wave phase across the $\mathrm{L}$ shells, respectively:

$C(L)=\exp \left(-\frac{\left(L-L_{\mathrm{pos}}\right)^{2}}{2 \sigma^{2}}\right) \cdot \exp (i \Theta)$.

The parameter $L_{\text {pos }}$ marks the position of maximum amplitude and phase jump. $L_{\text {pos }}$ cannot be determined directly from the observations as the RTI plot indicates only the apparent motion of the phase jump, but not its actual 


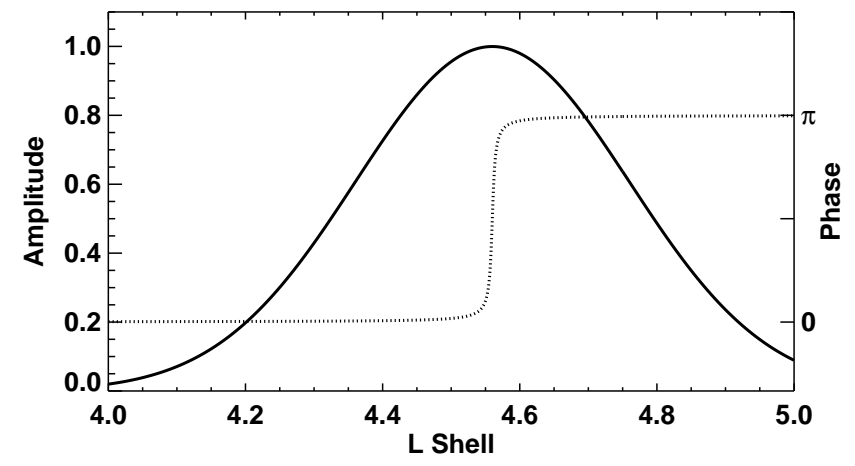

Fig. 11. Radial profile of the wave amplitude (thick line) and phase shift (dotted line).

position $L_{\text {pos. }}$. This position can be determined comparing modeled and observed time series. The radial width of the wave field is represented by $\sigma$. The distribution of the phase is modeled by $\Theta=(\pi / 2)+\arctan \left[w\left(L-L_{\text {pos }}\right)\right]$, where $w$ affects the width of the region within which the phase changes from $0^{\circ}$ to $180^{\circ}$. With these assumptions and the free model parameter $L_{\text {pos }}, \sigma$ and $w$ we generated in trial-and-error manner a series of pulsation wave fields. For $L_{\mathrm{pos}}=4.56, w=300 R_{E}^{-1}, \sigma=0.05 R_{E}$ for the first wave packet and $\sigma=0.2 R_{E}$ for the second one we found the best agreement between observed and modeled $b_{r}$ component, respectively their instantaneous amplitude. The radial distributions of wave amplitude and phase based on this set of parameters are displayed in Fig. 11 and the comparison between the observed and modelled signal is shown in Fig. 12.

The correspondence is evident in main features of the observed amplitude modulation, such as time and magnitude of the amplitude maxima of both wave packets (Fig. 12). Furthermore the modeled signal is capable to explain some detailed structure in the first wave packet (07:50-08:00 UT) of the spacecraft $\mathrm{C} 2, \mathrm{C} 3$ and $\mathrm{C} 4$ observation, which is caused by crossing the phase jump at $L=4.56$. However, some other details are not reproduced, e.g. the small-scale structure of the first wave packet seen by spacecraft $\mathrm{C} 1$ and in particular the narrow amplitude peaks at the beginning of the second wave packet visible in all four satellites. We speculate that the latter discrepancy between model and observation is a consequence of the assumed temporal amplitude modulation. Apparently modeling $b_{0}(t)$ by Gauss-functions fails to satisfy the real temporal evolution of a field line oscillation at least at the beginning of its lifetime. Nevertheless, modeling resulted in a value $L_{\mathrm{pos}}=4.56$ for the position of the excited field line.

\subsection{Poloidal Alfvén resonator}

The excited field line bundle is located in the outer edge of the plasmapause as inspection of measurements of the space-

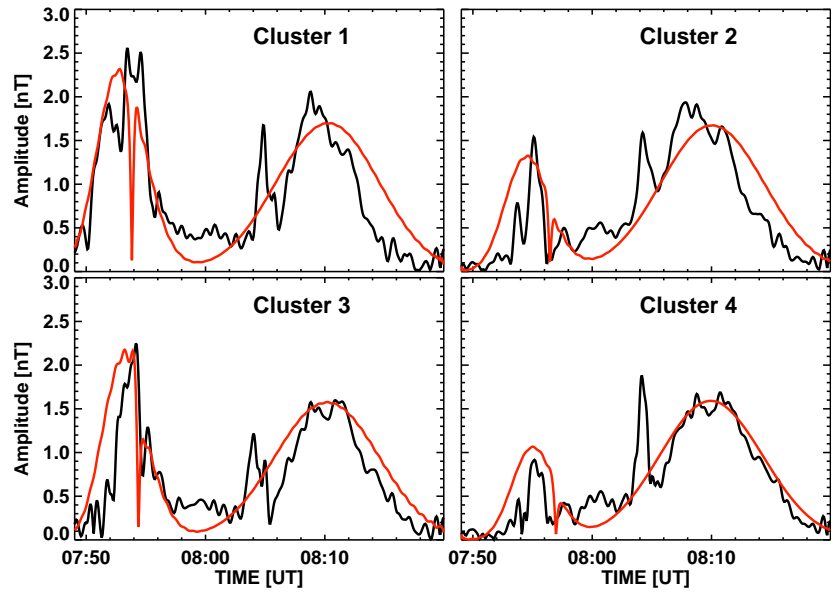

Fig. 12. Instantaneous amplitudes of the radial magnetic field component $b_{r}$ : observed signal (black) and modeled signal (red).

craft potential shows (Fig. 2). This outer region is suitable for the existence of an Alfvén resonator that confines the wave field by two poloidal turning points $L_{P}$ (see Sect. 1). On account of this we compare the assumed radial amplitude distribution and the observed frequency $f_{\mathrm{obs}}=23 \mathrm{mHz}$ of the pulsation with the radial profile of the the theoretically expected field line eigenfrequencies $\Omega_{P}$ and $\Omega_{T}$ (Fig. 14).

The theoretically expected toroidal eigenfrequency $\Omega_{T}$ is estimated using the time of flight method (Warner and Orr, 1979):

$\Omega_{T}=\frac{2 \pi N}{T_{A}} ; \quad T_{A}=2 \int_{S}^{N} \frac{d s}{v_{A}}$,

where $N$ is the longitudinal harmonic, $d s$ an increment of length along the field line and the integration limits are the northern and southern ionosphere. The Alfvén velocity $v_{A}(s)$ along the field line is given by $v_{A}(s)=B(s) / \sqrt{\mu_{0} \rho(s)}$. The magnetic field $B(s)$ is obtained from the Tsyganenko 96 model (Tsyganenko and Stern, 1996) for the given storm time index $D_{s t}=-7 \mathrm{nT}$, the dynamic pressure of the solar wind $p_{\mathrm{dyn}}=2.6 \mathrm{nPa}$ and $\mathrm{z}$-component of the interplanetary magnetic field $b_{z, S W}=5.0 \mathrm{nT}$. The distribution of the plasma density along field lines is assumed to follow the power law $\rho(s)=\rho_{e q}\left(L R_{E} / r\right)^{\alpha}$, where $\alpha=2$ is typical for the vicinity of the plasmapause (e.g. Goldstein et al., 2001). From the modeled electron density distribution $n_{e q}$ (Carpenter and Anderson, 1992) we receive the plasma mass density $\rho_{e q}=n_{e q} m_{\text {corr }} m_{p}$, where $m_{p}$ is the proton mass. Using a mass correction factor of $m_{\text {corr }}=2$ considers that the plasma near the plasmapause is composed of protons and a significant part of heavier ions such as $\mathrm{He}^{+}, \mathrm{O}^{+}$and $\mathrm{O}^{++}$(e.g. Takahashi et al., 2004).

The poloidal eigenfrequency $\Omega_{P}$ is computed using the numerical method based on the WKB approximation as described in Klimushkin et al. (2004). It requires information 

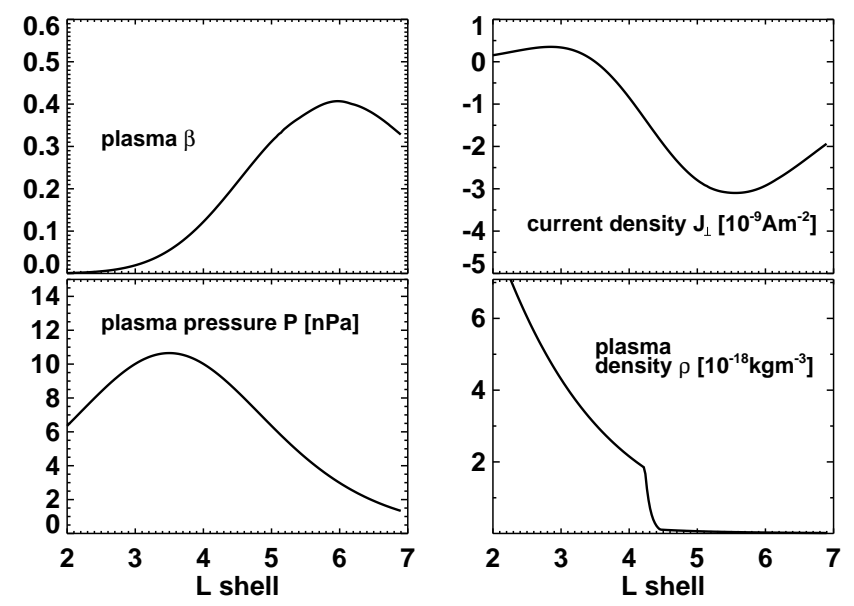

Fig. 13. Assumed plasma background conditions. The plasma density is calculated from modeling the electron density. Plasma pressure, $\beta$ and current density are assumed for quiet geomagnetic activity.

about plasma $\beta$, plasma pressure $P$ and strength of the ring current $J_{\perp}$ in the area of wave activity. At a low level of magnetospheric disturbance typical radial distributions of these quantities are assumed as displayed in Fig. 13 (Lui and Hamilton, 1992; Klimushkin et al., 2004).

In Fig. 14 the radial variation of eigenfrequencies $\Omega_{P}$ and $\Omega_{T}$ are compared with the modeled amplitude profile of the wave mode at time $t_{4}$ of its maximum extension. The poloidal eigenfrequency is slightly larger than the toroidal one which is a necessary condition for the existence of the Alfvén resonator (e.g. Leonovich and Mazur, 1995; Klimushkin et al., 2004). The poloidal turning points defined by $f_{\text {obs }}=f_{P}$ are identified at field lines $L=4.4$ and $L=5.0$ leading to a total width of the poloidal wave resonator of $0.6 R_{E}$. The modeled radial extend of the wave activity region is of the same size. The shift of the maximum of the amplitude distribution against the extremum of the eigenfrequency distribution is due to the asymmetry of the latter. We conclude that the wave field is confined in a poloidal Alfvén resonator at the outer edge of the dayside plasmapause.

The observed phase jump can be easily explained taking into account the radial localization and the rapid azimuthal variation $b_{r} \propto \exp (i m \Psi)$. This results in a radially localized polarization current causing a magnetic field perturbation sheared in radial direction (Klimushkin et al., 2004). However, two other processes are capable to explain the existence of a phase jump in magnetic field line oscillations: Field line resonance and phase mixing. The classical process of field line resonance causes the onset of purely toroidal polarized Alfvén waves and, accordingly, can be excluded as origin of the phase jump, as the observed wave is mainly poloidally polarized. Phase mixing of adjacent field lines is inappropriate as well to explain the phase jump, because the wave

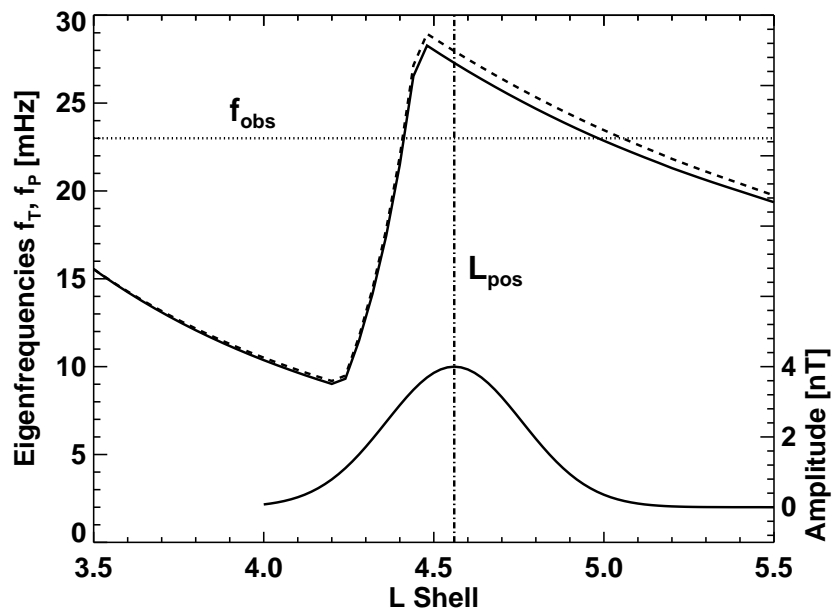

Fig. 14. Radial profiles of wave amplitude at time $t_{4}$ and toroidal (solid line) and poloidal (dashed line) field line eigenfrequencies, each for the second longitudinal harmonic. The dotted line marks the observed frequency $f_{\text {obs }}=23 \mathrm{mHz}$, the dot-dashed line marks the $\mathrm{L}$ shell of maximum amplitude and phase jump $L_{\mathrm{pos}}=4.56$.

packet is localized near the maximum of field line eigenfrequencies $\Omega_{P}(L)$, where the gradient $d \Omega_{P} / d L$ is small.

\section{Conclusions}

The simultaneous observation of magnetic and electric field data by the four Cluster satellites allows a detailed distinction between spatial and temporal characteristics of field line oscillations. For that purpose new representations of magnetic field data have been developed and applied to the observation of a mainly poloidal polarized standing field line oscillation with an observed frequency of $f_{\text {obs }}=23 \mathrm{mHz}$.

The pulsation analyzed was detected by the four Cluster satellites in the time interval 7 August 2003 between 07:46 and 08:20 UT and has been localized close to the magnetic equatorial plane at field lines between $L=4.42$ and $L=4.70$. Comparing the plasmapause position, obtained from a dynamical simulation of the electron density, with the spacecraft orbit exhibited that the pulsation was excited at the outer edge of the boundary between plasmasphere and magnetosphere.

The spatial separation of the spacecraft has given us the possibility to determine the phase differences between signals of pairs of satellites in order to estimate the azimuthal wave number $m$ of the pulsation. We have found $m \approx 155$ and consequently, a poloidal Alfvén wave with $m \gg 1$ has been observed.

A two wave packet structure of the pulsation has been detected in the instantaneous amplitude of the magnetic field oscillation featuring differences between both parts in terms of the wave polarization. In the first wave packet poloidal and 
toroidal magnetic field component have almost the same amplitude, whereas the second wave packet is purely poloidal for the most part. At first view this observation seems to be inconsistent with the theoretically expected evolution of a standing Alfvén wave which predicts a change in wave polarization from poloidal to toroidal (e.g. Mann and Wright, 1995; Mager and Klimushkin, 2006). However, displaying the instantaneous amplitude of the magnetic field oscillation in the LD coordinate system has elucidated a decrease of the wave amplitude in time at the same field lines for the first wave packet. Consequently, we have concluded that the first wave packet has been detected in a late stage of its temporal evolution when the amplitude of the toroidal component becomes comparable to the poloidal one. Wave damping due to dissipation at the ionosphere prevents that the wave field is observed in the status of purely toroidal polarization. The second wave packet has to be interpreted as a new excitation of a standing wave at the same field lines.

Modeling spacecraft observations crossing a double excited standing field line oscillation has confirmed and improved the knowledge about the above discussed spatial and temporal features of the detected wave field. It was shown that the wave is localized in radial direction with maximum of amplitude and phase jump located at $L=4.56$. The spatial extend of the wave field is around $0.6 R_{E}$. Location and size of the wave field was compared with the radial profile of theoretically expected toroidal and poloidal field line eigenfrequencies. We have suggested that the wave field is radially confined at the outer edge of the plasmapause between two L shells defined by $f_{\mathrm{obs}}=f_{P}$ acting as wave turning point. For that reason this specific region is believed to exhibit suitable conditions for the excitation of poloidal Alfvén waves (e.g. Klimushkin, 1998a).

The radial magnetic field oscillation observed in the four Cluster satellites has been combined to a RTI representation (Fig. 9) which has clearly exposed a change in the wave phase by $180^{\circ}$ in the radial direction. This phase jump is interpreted as a result of the localization of the wave field at the maximum of the radial amplitude distribution (Klimushkin et al., 2004).

A former case study by Schäfer et al. (2007) has dealt with a radially trapped poloidal ULF pulsation inside the dayside plasmapause using similar analysis method as the present work. Both studies have shown that spatial and temporal properties of the analyzed wave fields are in good agreement with main features predicted by the theoretical framework of Leonovich and Mazur (1990, 1993, 1995), Klimushkin et al. (2004) and Mager and Klimushkin (2006). However, some details of this framework concerning high- $m$ waves are not reported until now, neither in ground based data nor in space, e.g. clear indications for a transformation of wave polarization from poloidal to toroidal in time. Consequently, further investigation on ULF wave activity near the plasmapause are necessary to improve our knowledge on the spatio-temporal structure of magnetospheric Alfvén waves.
Acknowledgements. This work was financially support by the German Ministerium für Wirtschaft und Technologie and the Deutsches Zentrum für Luft- und Raumfahrt under contract 50OC0103. The work was supported by INTAS grant 05-1000008-7978. The work of P. N. Mager was also supported by RFBR grant 07-05-00185.

Topical Editor I. A. Daglis thanks W. Allan and another anonymous referee for their help in evaluating this paper.

\section{References}

Allan, W.: Phase variation of ULF pulsations along the geomagnetic field-line, Planet. Space Sci., 30, 339-346, 1982.

Allan, W., Poulter, E. M., Glassmeier, K. H., and Nielsen, E.: Ground magnetometer detection of a large m Pc5 pulsation observed with the STARE radar, J. Geophys. Res., 88, 183-188, 1983.

Allan, W., Poulter, E. M., and White, S. P.: Hydromagnetic wave coupling in the magnetosphere-Plasmapause effects on impulseexcited resonances, Planet. Space Sci., 34, 1189-1200, doi:10. 1016/0032-0633(86)90056-5, 1986.

Balogh, A., Carr, C. M., Acuna, M. H., and Dunlop, M. W.: The Cluster magnetic field investigation: overwiew of in-flight perfomance and initial results, Ann. Geophys., 19, 1207-1217, 2001, http://www.ann-geophys.net/19/1207/2001/.

Carpenter, D. L. and Anderson, R. R.: An ISEE/Whistler model of equatorial electron density in the magnetosphere, J. Geophys. Res., 97, 1097-1108, 1992.

Chen, L. and Cowley, S. C.: On field line resonances of hydromagnetic Alfven waves in dipole magnetic field, Geophys. Res. Lett., 16, 895-897, 1989.

Chi, P. J. and Russell, C. T.: Phase skipping and Poynting flux of continuous pulsations, J. Geophys. Res., 103, 29479-29492, 1998.

Denton, R. E. and Vetoulis, G.: Global poloidal mode, J. Geophys. Res., 103, 6729-6740, doi:10.1029/97JA03594, 1998.

Engebretson, M., Glassmeier, K.-H., Stellmacher, M., Hughes, W. J., and Lühr, H.: The dependence of high-latitude Pc5 wave power on solar wind velocity and on the phase of high-speed solar wind streams, J. Geophys. Res., 103, 26 271-26384, 1998.

Engebretson, M. J. and Cahill, L. J.: Pc5 pulsations observed during the June 1972 geomagnetic storm, J. Geophys. Res., 86, 56195631, doi:10.1029/0JGREA0000860000A7005619000001, 1981.

Engebretson, M. J., Zanetti, L. J., Potemra, T. A., and Acuna, M. H.: Harmonically structured ULF pulsations observed by the AMPTE CCE magnetic field experiment, Geophys. Res. Lett., 13, 905-908, 1986.

Eriksson, P. T. I., Blomberg, L. G., and Glassmeier, K.-H.: Cluster satellite observations of $\mathrm{mHz}$ pulsations in the dayside magnetosphere, Adv. Space Res., 23, 2679-2685, doi:10.1016/j.asr.2005. 04.103, 2005.

Escoubet, C. P., Schmidt, R., and Goldstein, M. L.: Cluster - Science and Mission Overview, Space Sci. Rev., 79, 11-32, 1997.

Fenrich, F. R., Samson, J. C., Sofko, G., and Greenwald, R. A.: ULF high- and low-m field line resonances observed with the Super Dual Auroral Radar Network, J. Geophys. Res., 100, $21535-$ $21548,1995$.

Fujita, S., Glassmeier, K. H., and Kamide, K.: MHD waves generated by the Kelvin-Helmholtz instability in a nonuniform mag- 
netosphere, J. Geophys. Res., 101, 27 317-27 326, doi:10.1029/ 96JA02676, 1996.

Glassmeier, K. H.: Magnetometer array observations of a giant pulsation event, J. Geophys., 48, 127-138, 1980.

Glassmeier, K. H.: On the influence of ionospheres with nonuniform conductivity distribution on hydromagnetic waves, J. Geophys., 54, 125-137, 1984.

Glassmeier, K. H.: Reconstruction of the ionospheric influence on ground-based observations of a short-duration ULF pulsation event, Planet. Space Sci., 36, 801-817, doi:10.1016/ 0032-0633(88)90086-4, 1988.

Glassmeier, K. H., Lester, M., Mier-Jedrzejowicz, W. A. C., Green, C. A., Rostoker, G., Orr, D., Wedeken, U., Junginger, H., and Amata, E.: Pc5 pulsations and their possible source mechanisms - A case study, J. Geophys., 55, 108-119, 1984a.

Glassmeier, K. H., Volpers, H., and Baumjohann, W.: Ionospheric Joule dissipation as a damping mechanism for high latitude ULF pulsations: Observational evidence, Planet. Space Sci., 32, 1463-1466, 1984b.

Glassmeier, K. H., Othmer, O., Cramm, R., Stellmacher, M., and Engebretson, M.: Magnetospheric Field Line Resonances: A Comparative Planetology Approach, Surv. Geophys., 20, 61109, 1999.

Goldstein, J., Denton, R. E., Hudson, M. K., Miftakhova, E. G., Young, S. L., Menietti, J. D., and Gallagher, D. L.: Latitudinal density dependence of magnetic field lines inferred from Polar plasma wave data, J. Geophys. Res., 106, 6195-6202, doi:10. 1029/2000JA000068, 2001.

Gustafsson, G., André, M., Carozzi, T., Eriksson, A. I., Fälthammar, C.-G., Grard, R., Holmgren, G., Holtet, J. A., Ivchenko, N., Karlsson, T., Khotyaintsev, Y., Klimov, S., Laakso, H., Lindqvist, P.-A., Lybekk, B., Marklund, G., Mozer, F., Mursula, K., Pedersen, A., Popielawska, B., Savin, S., Stasiewicz, K., Tanskanen, P., Vaivads, A., and Wahlund, J.-E.: First results of electric field and density observations by Cluster EFW based on initial months of operation, Ann. Geophys., 19, 1219-1240, 2001 , http://www.ann-geophys.net/19/1219/2001/.

Hasegawa, A.: Drift Mirror Instability in the Magnetosphere, Phys. Fluids, 12, 2642-2650, 1969.

Hughes, W. J.: The effect of the atmosphere and ionosphere on long period magnetospheric micropulsations, Planet. Space Sci., 22, 1157-1172, doi:10.1016/0032-0633(74)90001-4, 1974.

Hughes, W. J. and Southwood, D. J.: The screening of micropulsation signals by the atmosphere and ionosphere, J. Geophys. Res., 81, 3234-3240, 1976.

Kivelson, M. G. and Southwood, D. J.: Coupling of global magnetospheric MHD eigenmodes to field line resonances, J. Geophys. Res., 91, 4345-4351, 1986.

Klimushkin, D. and Mager, P. N.: The spatio-temporal structure of impulse-generated azimuthalsmall-scale Alfvén waves interacting with high-energy charged particles in the magnetosphere, Ann. Geophys., 22, 1053-1060, 2004, http://www.ann-geophys.net/22/1053/2004/.

Klimushkin, D., Mager, P. N., and Glassmeier, K. H.: Toroidal and poloidal Alfvén waves with arbitrary azimuthal wavenumbers in a finite pressure plasma in the Earth's magnetosphere, Ann. Geophys., 22, 267-287, 2004,

http://www.ann-geophys.net/22/267/2004/.
Klimushkin, D. Y.: Resonators for hydromagnetic waves in the magnetosphere, J. Geophys. Res., 103, 2369-2376, doi:10.1029/ 97JA02193, 1998a.

Klimushkin, D. Y.: Theory of azimuthally small-scale hydromagnetic waves in the axisymmetric magnetosphere with finite plasma pressure, Ann. Geophys., 16, 303-321, 1998b, http://www.ann-geophys.net/16/303/1998/.

Klimushkin, D. Y.: The propagation of high-m Alfvén waves in the Earth's magnetosphere and their interaction with highenergy particles, J. Geophys. Res., 105, 23 303-23 310, doi: 10.1029/1999JA000396, 2000.

Lee, D.-H. and Lysak, R. L.: Effects of azimuthal asymmetry on ULF waves in the dipole magnetosphere, Geophys. Res. Lett., 17, 53-56, 1990.

Leonovich, A. S. and Mazur, V. A.: The spatial structure of poloidal alfven oscillations of an axisymmetric magnetosphere, Planet. Space Sci., 38, 1231-1241, doi:10.1016/0032-0633(90) 90128-D, 1990.

Leonovich, A. S. and Mazur, V. A.: A theory of transverse small-scale standing Alfvén waves in an axially symmetric magnetosphere, Planet. Space Sci., 41, 697-717, doi:10.1016/ 0032-0633(93)90055-7, 1993.

Leonovich, A. S. and Mazur, V. A.: Magnetospheric resonator for transverse-small-scale standing Alfven waves, Planet. Space Sci., 43, 881-883, 1995.

Lui, A. T. Y. and Hamilton, D. C.: Radial profiles of quiet time magnetospheric parameters, J. Geophys. Res., 97, 19325-19332, 1992.

Mager, P. N. and Klimushkin, D. Y.: On impulse excitation of the global poloidal modes in the magnetosphere, Ann. Geophys., 24, 2429-2433, 2006, http://www.ann-geophys.net/24/2429/2006/.

Mann, I. R. and Wright, A. N.: Finite lifetimes of ideal poloidal Alfvén waves, J. Geophys. Res., 100, 23 677-23 686, doi:10. 1029/95JA02689, 1995.

McDiarmid, D. R. and Allan, W.: Simulation and analysis of auroral radar signatures generated by a magnetospheric cavity mode, J. Geophys. Res., 95, 20 911-20 922, 1990.

McIlwain, C. E.: Magnetic Coordinates, Space Sci. Rev., 5, 585598, 1966.

Ozeke, L. G., Mann, I. R., and Mathews, J. T.: The influence of asymmetric ionospheric Pedersen conductances on the fieldaligned phase variation of guided toroidal and guided poloidal Alfvén waves, J. Geophys. Res. (Space Physics), 110, A08210, 1-16, doi:10.1029/2005JA011167, 2005.

Pierrard, V. and Cabrera, J.: Comparisons between EUV/IMAGE observations and numerical simulations of the plasmapause formation, Ann. Geophys., 23, 2635-2646, 2005, http://www.ann-geophys.net/23/2635/2005/.

Pierrard, V. and Lemaire, J. F.: Development of shoulders and plumes in the frame of the interchange instability mechanism for plasmapause formation, Geophys. Res. Lett., 31, 5809-5813, doi:10.1029/2003GL018919, 2004.

Poulter, E. M., Nielsen, E., and Potemra, T. A.: Field-aligned currents associated with Pc 5 pulsations - STARE and TRIAD observations, J. Geophys. Res., 87, 2331-2336, 1982.

Samson, J. C., Jacobs, J. A., and Rostoker, G.: Latitude-Dependenct Characteristic of Long-Period Geomagnetic Micropulsations, J. Geophys. Res., 76, 3675-3683, 1971. 
Schäfer, S., Glassmeier, K. H., Eriksson, P. T. I., Pierrard, V., Fornaçon, K. H., and Blomberg, L. G.: Spatial and temporal characteristics of poloidal waves in the terrestrial plasmasphere: a CLUSTER case study, Ann. Geophys., 25, 1011-1024, 2007, http://www.ann-geophys.net/25/1011/2007/.

Sciffer, M., Waters, C., and Menk, F.: Propagation of ULF waves through the ionosphere: Inductive effect for oblique magnetic fields, Ann. Geophys., 22, 1155-1169, 2004, http://www.ann-geophys.net/22/1155/2004/.

Sciffer, M. D., Waters, C. L., and Menk, F. W.: A numerical model to investigate the polarisation azimuth of ULF waves through an ionosphere with oblique magnetic fields, Ann. Geophys., 23, 3457-3471, 2005, http://www.ann-geophys.net/23/3457/2005/.

Singer, H. J. and Kivelson, M. G.: The latitudinal structure of Pc 5 waves in space - Magnetic and electric field observations, J. Geophys. Res., 84, 7213-7222, 1979.

Singer, H. J., Hughes, W. J., and Russell, C. T.: Standing hydromagnetic waves observed by ISEE 1 and 2 - Radial extent and harmonic, J. Geophys. Res., 87, 3519-3529, 1982.

Southwood, D. J.: Some features of field line resonances in the magnetosphere, Planet. Space Sci., 22, 483-491, 1974.

Southwood, D. J. and Kivelson, M. G.: Charged particle behavior in low-frequency geomagnetic pulsations. II - Graphical approach, J. Geophys. Res., 87, 1707-1710, 1982.

Southwood, D. J., Dungey, J. W., and Etherington, R. J.: Bounce resonant interaction between pulsations and trapped particles, Planet. Space Sci., 17, 349-361, 1969.

Takahashi, K. and Anderson, B. J.: Distribution of ULF energy (f is less than $80 \mathrm{mHz}$ ) in the inner magnetosphere - A statistical analysis of AMPTE CCE magnetic field data, J. Geophys. Res., 97, 10751-10773, 1992.
Takahashi, K., Denton, R. E., Anderson, R. R., and Hughes, W. J.: Frequencies of standing Alfvén wave harmonics and their implication for plasma mass distribution along geomagnetic field lines: Statistical analysis of CRRES data, J. Geophys. Res. (Space Physics), 109, 1-15, doi:10.1029/2003JA010345, 2004.

Tamao, T.: Transmission and coupling resonance of hydrodynamic disturbances in the non-uniform Earth's magnetosphere, Sci. Rep. Tohoku Univ., Ser 5, 17, 1965.

Tsyganenko, N. A.: Modeling the Earths magnetospheric magnetic field confined within a realistic magnetopause, J. Geophys. Res., 100, 5599-5612, 1995.

Tsyganenko, N. A. and Stern, D. P.: Modeling the global magnetic field of the large-scale Birkeland current systems, J. Geophys. Res., 101, 27 187-27 198, 1996.

Vetoulis, G. and Chen, L.: Global structures of Alfven-ballooning modes in magnetospheric plasmas, Geophys. Res. Lett., 21, 2091-2094, 1994.

Vetoulis, G. and Chen, L.: Kinetic theory of geomagnetic pulsations 3. Global analysis of drift Alfvén-ballooning modes, J. Geophys. Res., 101, 15 441-15 456, doi:10.1029/96JA00494, 1996.

Walker, A. D. M., Greenwald, R. A., Stuart, W. F., and Green, C. A.: STARE auroral radar observations of Pc 5 geomagnetic pulsations, J. Geophys. Res., 84, 3373-3388, 1979.

Warner, M. R. and Orr, D.: Time of flight calculations for high latitude geomagnetic pulsations, Planet. Space Sci., 27, 679-689, doi:10.1016/0032-0633(79)90165-X, 1979.

Wright, A. N., Allan, W., Elphinstone, R. D., and Cogger, L. L.: Phase mixing and phase motion of Alfvén waves on tail-like and dipole-like magnetic field lines, J. Geophys. Res., 104, 10159 $10176,1999$. 